\title{
Edge IoT Networking
}

\author{
Majed Mohaia Alhaisoni \\ Department of Computer Sceince \\ University of Ha'il, Ha'il \\ Saudi Arabia
}

\begin{abstract}
Data transmission has witnessed a new wave of emerging technologies such as IoT. This new way of communication could be done through smart communication such as smart sensors and actuators. Thus, data traffic keeps traversing to the main servers in order to accomplish the tasks at the sensors side. However, this way of communication has encountered certain issues related to network due to the nature of routing forth and back from the end users to the main servers. Subsequently, this incurs high delay and packet loss which successively degrades the overall Quality of Service (QoS). On the other hand, the new way of data transmission, which is called "edge IoT network", has not only helped on reducing the load over the network but also made the nodes to be more self-manage at the edge. However, this approach has some limitations due to the power consumption and efficiency, which would lead to node failure and data loss. Therefore, this paper presents a new model of combining network science and computer network in order to enhance the edge IoT efficiency. Simulation results have shown a clear evidence in improving the efficiency, communicability, degree, and overall closeness.
\end{abstract}

Keywords-Edge IoT; network centrality; communicability; degree; closeness

\section{INTRODUCTION}

In the past few decades, the advances in technology is increasing very rapidly where end terminals have become wellspread over the network. The massive development in technologies, such as wireless communications and mobiles have created a platform for people to exchange information easily. Moreover, lightweight smart devices are going up, not only that but also daily life has become more interconnected across things [1]. Hence, wireless sensors network (WSN) is one of the most promising and convenient for data gathering, mainly at the era of Internet of things (IoT) [2]. However, such heterogeneous in data communication and gathering shall bring more burden over the network. Consequently, big data is more generated which needs careful consideration in terms of data classification and analysis [3].

IoT is nowadays being active technology where things are all interconnected at home, road, and buildings. Hence, actuators, sensors are commonly used to interact between devices for data transmission and processing [4]. However, since sensors are tied up with life span due to the battery lifetime and energy, this cause a critical hurdle for such type of communication to be more spread and utilized in communication and networking [5]. Therefore, various techniques were proposed in literature to address the issues related to power consumption and life span of WSN such as [6][7][8]. On the other hand, WSN has faced some issues and limitations on communicating with servers and cloud due to the sensitivity of data which is intolerable of low data rate and high latency [9]. Thus, these two key factors are very critical for IoT where massive computation and high traffic needs to be transferred timely to the end users. Henceforward, Edge IoT paradigm has become the most interesting way of communication which could help on alleviating previous highlighted issues related to communication between WSN and cloud [10]. In this approach, technology is moving from centralized cloud into distributed Edge nodes at the network. Therefore, nodes act as a client-server simultaneously which acts on receiving requests and processing at the same time with no need of interconnecting with the cloud [11]. This new way of interconnection has undoubtedly improved the latency and efficiency. However, edge devices encounter limitations of computing resources and life span at the network, which cause data loss and halted issues for data transmission, and processing.

This paper looks at the efficiency and load distribution issues at the edge IoT network. Hence, a new algorithm has been proposed to overcome these limitations. Thus, It combines network science and computer network in order to get the most use of it. Therefore, since edge devices are spread at the network edge, It is very important to consider the network centrality and load distribution. In other words, devices at the edge are not supposed to be constantly interconnected to many devices and serving them concurrently. This would easily abuse resources and make the nodes in a risk of failure at any time, furthermore, date rate would also be affected which shall degrade the QoS and response time for request by other end users. Results have shown a clear evidence in enhancing the centrality, communicability, and overall closeness.

\section{RELATED WORK}

Edge computing has gained more attention recently since it is still evolving as a technology paradigm. Therefore, Nokia and IBM have launched the first edge computing at early 2013. Following that, auspices of the European Telecommunications Standards Institute (ETSI) has introduced a mobile edge computing. Moreover, in 2015 opencomputingedge.org was launched by Vodafone, Intel, Huwai in cooperation with Carnegie Mellon University (CMU) [12]. On the other hand, research has focused on studying different paradigms of edge IoT, and discussed various issues and limitation on edge IoT. In [13] edge computing has been proved that it is very useful as a local server, mainly at complex core network. Moreover, a third-party application was implemented as a local server and shown a better performance. 
Another study in [14] has proposed a game theory approach to offload the computation in distributed edge IoT rather than cloud, their results have shown that IoT is more effective on computational rather cloud based. Moreover, device-to-device at edge has been proposed in [15] where they have clearly shown that offloading resources at edge enhance the efficiency. Furthermore, placing cloudlet at edge approach has been introduced by [16] where they have traded-off between the cost of placing these cloudlets and the gained end-to-end delay. In their approach, workload is assigned to the best-located cloudlet in order to reduce the response time for users' requests. Additionally, resources allocation at edge IoT is discussed in [17] where they have shown how limited resources can be optimized with consideration of plan capacity at the edge. However, existing work has analyzed the disadvantages of moving data from cloud to the end users where latency is one of the main hurdles in such approach. Therefore, in [18] authors have proposed a cloudlet to run the big data instead of adopting cloud model. This approach has shown an effective way of enhancing the communication between end users and big data applications.

Furthermore, GigaSight model has been introduced by [19] in order to minimize the latency between end users and cloudlet in processing the video while the bandwidth is reserved within the core network. In addition to previous studies, authors in [20] have proposed a mobile edge IoT where Base station is connected to a fog node which act locally by handling the data without the need to connect remotely to cloud. However, in order to alleviate the overhead on forwarding the packets among nodes, SDN based was utilized on top of fog nodes. This approach has been effective on reducing the end-to-end delay. Moreover, end -to-end delay reduction among end users is considered by [21] where green energy is employed in order to migrate VMs to cloudlet and assures a minimal latency between users.

Power consumption for edge IoT is another issue facing this approach of communication. Hence, authors in [22] has proposed various techniques in smart devices in order to prolong the energy life of edge nodes. Moreover, energy optimization is considered in [23] where profiling scheme is proposed in order to monitor the usage trends in mobile applications which would consequently lead to power optimization. On the other hand, mobile devices are equipped with numerous amount of applications to serve different purposes, these apps consume high utilization of battery which affect hereafter the overall power consumption. Therefore, various techniques were proposed in [24] to ensure mobile optimization at the network edge. Additionally, mobile efficiency is discussed in [25] where software defined network is introduced to enhance the overall efficiency by considering two different components such as security and storage. Moreover, power optimization was also reviewed by [26] where a mathematical modelling was proposed to enhance the energy in mobile cloud but in a very large scale network. In addition to previous studies, authors in [27] has proposed a model to place various cloudlets across IoT in order to reduce the average end-to-end delay among end users. However, in line with their study, another work was proposed by [28] where they have introduced different techniques in order to place cloudlets in strategic locations with the aim to minimize the latency but with consideration of balancing the load among cloudlets to avoid overwhelming any of these cloudlets from failures which may lead to a collapse of network.

In contrast to the previous reviewed studies, this paper looks at the edge IoT efficiency and load distribution from network science perspective. Hence, network centrality plays a vital role on load distribution over the network. Therefore, the proposed algorithm defines carefully the nodes centrality at the edge, not only that but also load distribution is enhanced by introducing a reshuffling mechanism which ensures that nodes are not fully occupied by huge connections with other nodes. Thus, this work is in line with previous studies but with new techniques of combining two different science together to enhance edge IoT efficiency. The following section shall give more details of the proposed algorithm.

\section{PROPOSED METHOD}

Mobile edge computing is gaining more attention recently since it's the medium of current communication in most networking environment [29]. Therefore, mobile devices or scattered sensors, which are attached to devices, are forming such type of mobile edge network. This new way of communication has been utilized for solid purpose as discussed in related work section, which is mainly to avoid remote communication with cloud and resides servers on remote areas. However, due to some limitations in the current edge IoT environment, this paper proposes an algorithm, which combines, network science and computer network together to achieve the best optimum efficiency in edge IoT network. As mentioned earlier, mobile devices at the edge of network interact with each other which in returns contribute to the network and help IoT at the edge of not contacting the server for computation and content retrieval. However, since these devices and sensors contribute their power, bandwidth, budgeting, and computing resources. It is very logical to face issues on how to maintain these resources and assure sustainability and stability of the network. Moreover, dealing with high demand on traffic and data would lead to overload of sensors and devices at the edge, this shall cause depletion to these nodes and network failures.

However, different from other previous discussed work, this algorithm is introduced to mainly look at efficiency issue at edge IoT. Therefore, a network science methodology to enhance the load distribution and power efficiency is proposed. Thus, from network science perspective, network centrality metric [30] was the key to measure the efficiency of a dense network at the edge. Thereafter, the communicability [31], which measures the interconnectivity among nodes at the network edge is gauged. In return, after evaluating the communicability values for those nodes located at the edge, the algorithm takes an additional step; to rank these nodes based upon the communicability values in a top-down approach.

Subsequently, the algorithm re-measures the network centrality on those nodes interacting with each other at the edge and keeps reshuffling in order to distribute the load over the these nodes with solid centrality over them. However, the proposed algorithm is also considering the overall degree and closeness on examined nodes. These two metrics play a vital 
role on the locality of selection of nodes at the edge. Hence, it helps on maintaining nearby nodes instead of interacting with remote devices where traffic travers core network to reach up to destination. This does not only degrade the bandwidth but also affects the overall quality of delivered content. Another critical metric, which could be affected by neglecting the closeness of nodes at the edge, is end-to-end delay due to the random connection among the nodes. Following section shall give more details of implementation of this algorithm where various techniques are combined and introduced together to show the best optimum values of gained efficiency of the examined network.

\section{Evaluation Method}

In order to simulate the proposed algorithm, the Python ${ }^{1}$ has been adapted as a platform to build and implement a simulated an edge IoT scenario. It is also a high level language with various options of embedded libraries which gives a strong platform for network simulation. However, experimental work has been run over various network sizes randomly although nodes at the edges were carefully selected for execution of the proposed algorithm. Moreover, there are different types of models for topologies, therefore, The ErdösRényi model ${ }^{2}$ was used to generate the graphs. Hence, at the initial phase, $\mathrm{N}$ nodes are generated with full distribution across nodes with budget for every node. At the startup phase, nodes are formed to represent a graph, which shows edge nodes. Afterwards, the algorithm runs a calculation for communicability to gauge the network centrality. This procedure gives a clear image of the conditions of nodes across network and shows the level of interconnection among them.

Thereafter, since the objective of this paper is to focus on edge nodes, the algorithm considers only the values of those nodes. Therefore, the algorithm organizes the edge nodes values as high and low values, hence, the communicability is measured regularly after each run, and values are ordered accordingly. Thus, the gained efficiency is calculated for these nodes and then carefully checks the edge nodes efficiency.

Furthermore, after a thorough analysis of the edge nodes values in terms of communicability and gained efficiency, the algorithm executes a reshuffling scenario, which is known as "rewiring". The aim of this operation is to enhance the load distribution by redirecting links from those edge nodes with high values to those nodes achieving low communicability values. However, It is essential to emphasize that this action takes place iteratively followed by a measurement of gained efficiency to gauge the level of load distribution among edge nodes. However, to ensure that the algorithm does not continue in a loop of reshuffling, an exit condition is defined. This condition is embedded in the algorithm where communicability values are not changing significantly anymore. Moreover, for validation purpose, the algorithm has been run over various sizes of nodes in order to examine the robustness of the algorithm over different conditions.
1) Network centrality: Network centrality is the main function, which gives an insight of the complexity of any type of network. Hence, it has been introduced in the proposed algorithm as of gauging the centrality metric [30]. It reflects as one of the main reference to measure the efficiency of any type of a dense network. Moreover, it captures the level of interconnection between entities, nodes, persons, and so on. In IoT, this plays a vital role where nodes are spread all over. Therefore, it is critical to measure the degree of communication between these nodes and pinpoint carefully the best value of centrality in the network.

2) Communicability: Another factor considered in the proposed algorithm is known as the communicability ${ }^{3}$. This refers to measuring the interconnectivity between nodes all over the network topology. In return, after evaluating the communicability values for all nodes, an additional step is taken by the algorithm, to rank the nodes in the network based upon the communicability, from top to low values.

3) Reshuffling: A new element introduced in the algorithm is "reshuffling". This mechanism acts on re-distributing the links among nodes according to the output of above (A\&B). Basically, in some network scenarios, it can be found that some edge nodes are overwhelmed by the amount of outgoing connections whereas other edge nodes are almost at level of depletion cause of having no activity in the network and not contributing the overall distribution. Hence, the main target of this technique is to balance the amount of connections among the edge nodes, taking in consideration that each edge node has at least one connection in the network and staying alive across the network life span. However, a trade-off between reshuffling and network centrality is considered carefully throughout the whole implementation of the algorithm. On the other hand, Quality of service is considered where the algorithm keeps checking packet loss, end-to-end delay metrics to make sure that the minimum level of QoE (Quality of Service) are meeting the minimum acceptable threshold, according to [32].

\section{RESUlTS AND DISCUSSION}

This section gives more insights on the output of the simulation of proposed algorithm. Therefore, in order to gauge the performance properly, two scenarios have been introduced. The first scenario represents the proposed algorithm (referred to as "with reshuffling"); whereas the second scenario was introduced to mimic a randomized scenario without any kind of self-adaptation of the WSN (referred to as "without reshuffling"). Moreover, various metrics have been introduced to show the strength of algorithm such as communicability, gained efficiency, closeness, degree, computational time, and number of iterations. These metrics are defined as follows:

\footnotetext{
1"Python." [Online]. Available: https://www.python.org

2“"Erdos Renyi models.pdf." https://en.wikipedia.org/wiki/ErdősRényi_model
} 
Communicability: This metric gives a clear understanding of the load distribution over the WSN in terms of power and energy. The lower values achieved in communicability, the better the load distribution is realized over the network.

Gained Efficiency: This metric is to show network efficiency and how the nodes are contributing to the overall network. It is interlinked with the overall communicability each time. In the proposed algorithm, gained efficiency (GE) is defined as follows:

$\mathrm{GE}=(\Delta / \max$ values $) * 100$

Where $\Delta$ is (MAX - the overall communicability value). The Max value in this case is 1 (depending on the metrics scale).

Degree: This metric shows of how many connections each node at the edge has. It gives an image of the performance of the proposed method in distributing the load at the edge, and helps on alleviating edge node from traffic overload.

Closeness: Since the nodes are spread at the edge, It is essential that nodes connect closely with those nodes close to each other. Therefore, since the proposed algorithm reshuffles the nodes and re-connects them to those nodes with low values of communicability, this procedure helps on distributing the load across edge.

Computational time: This metric is very valuable in measuring the robustness of the proposed algorithm against the randomised approach. Thus, as the network size goes up, this has drastic impact on the performance of showing complicated scenarios. Dealing with a large-scale network size has a significant impact on the performance of executing very complicated scenarios.

Fig. 1 indicates how the network load is distributed across edge IoT network. Thus, both scenarios have been run at the same conditions in terms of network size and connections. However, network graph is randomly generated at the start up, then, communicability is measured for those nodes at the edge only, afterwards, the proposed algorithm starts its process by reshuffling nodes per communicability values. Therefore, it is very obvious from Fig. 1 that proposed method is capable to achieve a steady decrease across various network size which supports the robustness of the proposed algorithm regardless of the network size. Moreover, both scenarios were repeated many times, the results shown here is the average and standard deviation of these runs. This gives meaningful and valid output of the examined scenarios. However, in terms of the performance of the proposed algorithm, It is very clear that communicability values across network size is showing steady decrease and this is an evidence that load and power is fully distributed among nodes at the edge. Therefore, results confirm that such behavior would help on supporting the trend of relying heavily on edge IoT instead of connecting with main servers, which has some limitations as highlighted earlier.

On the other hand, Fig. 2 shows another angle of testing the proposed algorithm, which is the gained efficiency. This metric depends heavily on the performance of the communicability, in other words, it is very linked with each other. Thus, it would not be possible to examine any proposed scenario separately from each other. Therefore, the results, which are achieved here, are consistent with the output highlighted in Fig. 1. Hence, both scenarios have been examined at the same conditions in terms of network size. However, It is obvious that the proposed algorithm is showing steady increase in efficiency, and by looking closely to Fig. 1, It can be claimed that this results is against the achieved communicability by the same algorithm which is an evidence that the lower communicability values are, the more efficiency gained accordingly. Furthermore, results give another line of confirmation that distributing the load at the edge network would be powerful to rely heavily on nodes at the edge, and saving energy and power since they contribute by their own computing resources. However, looking at the randomized scenario which is called "without reshuffling", It is obvious that gained efficiency is almost obsolete due to the randomness in connections, not only that but also, load is not distributed among nodes, and this is backed up by the results shown in Fig. 1 where the communicability values are steady line across various network size.

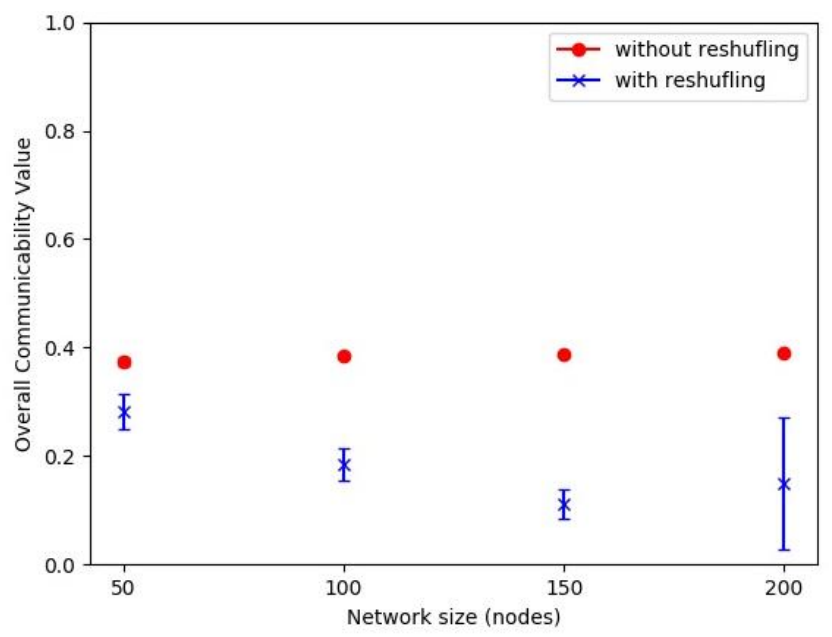

Fig. 1. Overall Communicability.

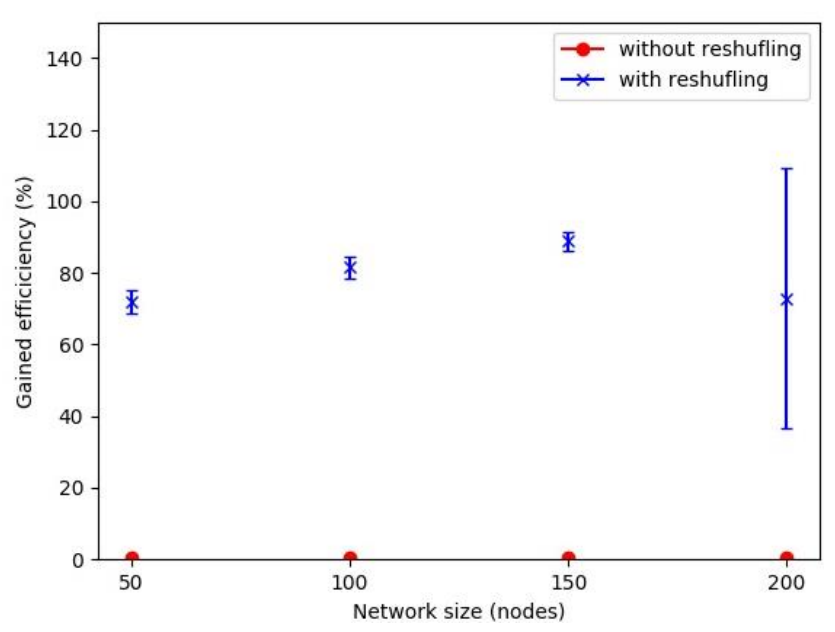

Fig. 2. Gained Efficiency. 
Another factor that plays a vital role on edge IoT network is the degree value. This metric has been selected to show the amount of connections that each node at the edge is handling. Hence, the lower connections mean that the higher load distribution is achieved across the edge. Therefore, Fig. 3 shows the degree level at the proposed algorithm "with reshuffling" against the scenario "without reshuffling". Subsequently, the results show that the embedded techniques have shown a steady decrease in degree level, and this is consistent with the previous highlighted results in Fig. 1 and 2. On other hand, the benchmarking scenario shows steady line where the number of connections at each run over various network size is almost the same. Thus, there is no consideration of load distribution as well as energy efficiency, which could affect the nodes at the edge and lead to battery depletion, which may incur network failure.

Furthermore, Fig. 4 gives another insight of the value of the proposed algorithm, which measures the closeness among connected nodes at the edge level. Hence, Fig. 4 shows a clear evidence that combining network science and computer network not only enhance the load distribution and efficiency but helps on the closeness among nodes. Hereafter, Fig. 4 indicated that the overall closeness is decreased steady against the network size, this is certainly an evidence that the proposed algorithm is performing efficiently across various conditions.

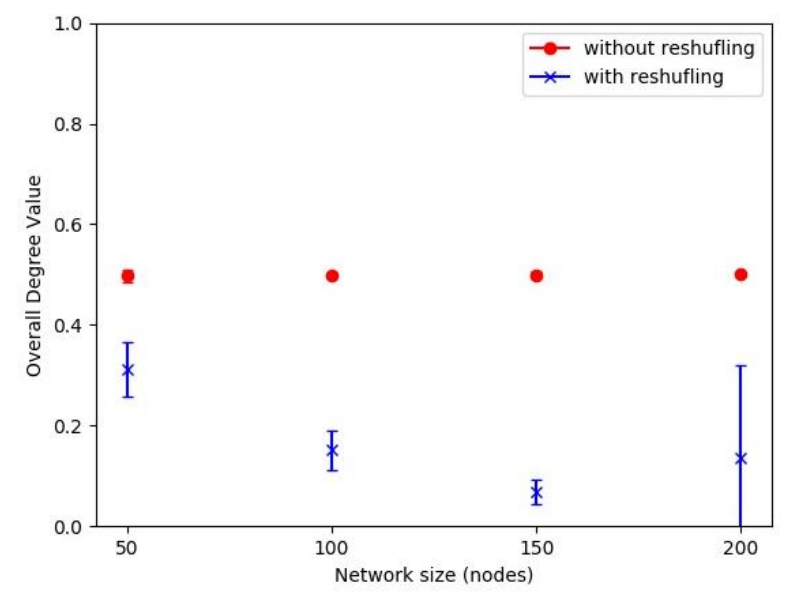

Fig. 3. Overall Degree.

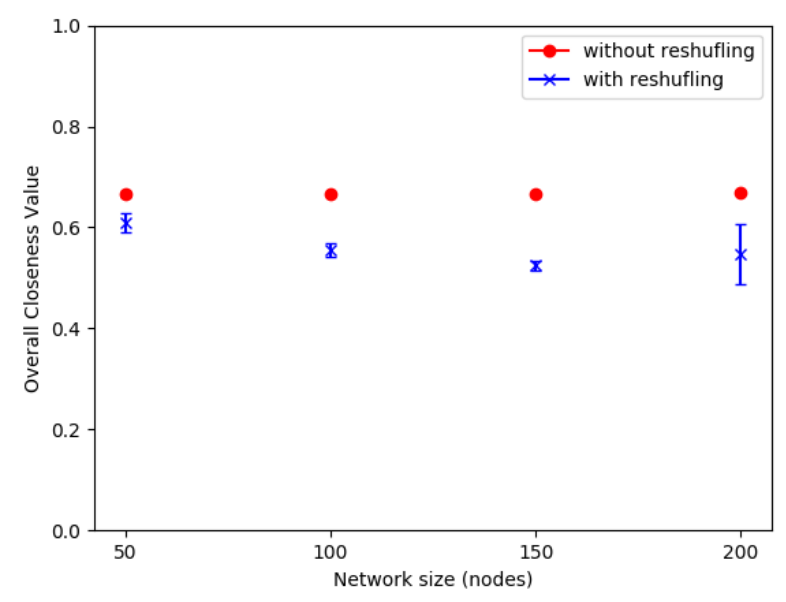

Fig. 4. Overall Closeness.

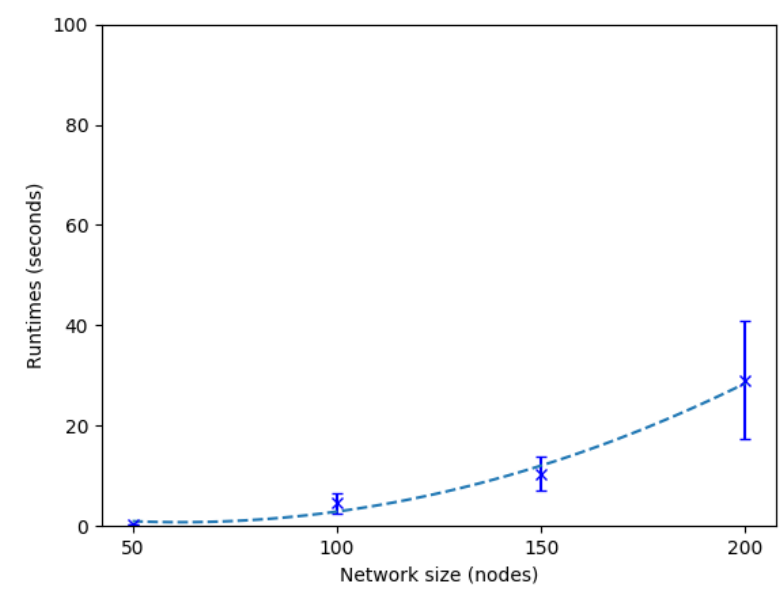

Fig. 5. Computational Time.

Computational time is another critical factor of the proposed algorithm, since it shows the robustness of the proposed approach. Therefore, dealing with a large-scale network size has a significant impact on the performance of executing very complicated scenarios. This metric was very valuable in measuring the computational efficiency of the proposed algorithm, in terms of running time and execution.

Fig. 5 indicates that the running time increases linearly against the network size. A careful examination of the amount of elapsed time necessary to run, execute, and achieve the best results over many runs, demonstrates that the algorithm performs very well despite the network size and the complexity of connections among the nodes.

\section{CONCLUSION}

Edge IoT is given more attention recently due to the advantages shown against traditional IoT paradigm, which relies heavily on connecting to reside servers at the main core network. Therefore, this paper presents an edge IoT scenario with combination of network science and computer network in order to show how such combination would lead to efficient utilization of edge IoT network. Results have clearly shown that edge IoT is very promising approach in IoT, with the consideration of load distribution and power efficiency. Hence, the proposed algorithm has shown robustness in terms of various metrics such as network centrality, communicability, degree, and closeness. Moreover, the proposed algorithm was compared to mimic a traditional scenario, which has no techniques to enhance the efficiency and, load distribution. Future work will focus more on QoS and QoE metrics by injecting a video streaming across network and examine the proposed algorithm over various condition of network.

REFERENCES

[1] J. Ren, Y. Zhang, R. Deng, N. Zhang, D. Zhang, and X. S. Shen, "Joint channel access and sampling rate control in energy harvesting cognitive radio sensor networks," IEEE Trans. Emerg. Top. Comput., vol. 7, no. 1, pp. 149-161, 2019.

[2] T. Saarikko, U. H. Westergren, and T. Blomquist, "The Internet of Things: Are you ready for what's coming?," Bus. Horiz., vol. 60, no. 5, pp. 667-676, 2017.

[3] M. Gayathri and K. Srinivas, "A Survey on mobile cloud computing architecture, applications and challenges," Int. J. Sci. Res. Eng. Technol., vol. 3, no. 6, pp. 1013-1021, 2014. 
[4] P. Bellavista, J. Berrocal, A. Corradi, S. K. Das, L. Foschini, and A. Zanni, "A survey on fog computing for the Internet of Things," Pervasive Mob. Comput., vol. 52, pp. 71-99, 2019.

[5] A. Pal and K. Kant, "NFMI: Connectivity for Short-Range IoT Applications," Computer (Long. Beach. Calif)., vol. 52, no. 2, pp. 63-67, 2019.

[6] Q. Wang, D. Lin, P. Yang, and Z. Zhang, "An Energy-Efficient Compressive Sensing-Based Clustering Routing Protocol for WSNs," IEEE Sens. J., vol. 19, no. 10, pp. 3950-3960, 2019.

[7] R. Yarinezhad, "Reducing delay and prolonging the lifetime of wireless sensor network using efficient routing protocol based on mobile sink and virtual infrastructure," Ad Hoc Networks, vol. 84, pp. 42-55, 2019.

[8] M. Chincoli and A. Liotta, "Self-learning power control in wireless sensor networks," Sensors (Switzerland), vol. 18, no. 2, pp. 1-29, 2018.

[9] A. A. Banka and R. N. Mir, "Current Big Data Issues and Their Solutions via Deep Learning: An Overview.," Iraqi J. Electr. Electron. Eng., vol. 14, no. 2, pp. 127-138, 2018.

[10] M. Amadeo, G. Ruggeri, C. Campolo, and A. Molinaro, "IoT services allocation at the edge via named data networking: From optimal bounds to practical design," IEEE Trans. Netw. Serv. Manag., vol. 16, no. 2, pp. 661-674, 2019.

[11] Y. Ai, M. Peng, and K. Zhang, "Edge computing technologies for Internet of Things: a primer," Digit. Commun. Networks, vol. 4, no. 2, pp. 77-86, 2018.

[12] M. Satyanarayanan, "The emergence of edge computing," Computer (Long. Beach. Calif)., vol. 50, no. 1, pp. 30-39, 2017.

[13] A. M. Khan and F. Freitag, "On Participatory Service Provision at the Network Edge with Community Home Gateways," Procedia Comput. Sci., vol. 109, pp. 311-318, 2017.

[14] S. Kim, "Nested game-based computation offloading scheme for Mobile Cloud IoT systems," Eurasip J. Wirel. Commun. Netw., vol. 2015, no. 1, 2015.

[15] B. Li, Z. Fei, J. Shen, X. Jiang, and X. Zhong, "Dynamic Offloading for Energy Harvesting Mobile Edge Computing: Architecture, Case Studies, and Future Directions," IEEE Access, vol. 7, pp. 79877-79886, 2019.

[16] Q. Fan and N. Ansari, "Cost Aware cloudlet Placement for big data processing at the edge," IEEE Int. Conf. Commun., pp. 1-6, 2017.

[17] F. Slim, F. Guillemin, and Y. Hadjadj-Aoul, "On virtual network functions' placement in future distributed edge cloud," Proc. 2017 IEEE 6th Int. Conf. Cloud Networking, CloudNet 2017, pp. 1-4, 2017.

[18] L. A. Tawalbeh, W. Bakheder, and H. Song, "A Mobile Cloud Computing Model Using the Cloudlet Scheme for Big Data Applications," Proc. - 2016 IEEE 1st Int. Conf. Connect. Heal. Appl. Syst. Eng. Technol. CHASE 2016, pp. 73-77, 2016.
[19] M. Satyanarayanan et al., "Edge analytics in the internet of things," IEEE Pervasive Comput., vol. 14, no. 2, pp. 24-31, 2015.

[20] X. Sun and N. Ansari, "EdgeIoT: Mobile Edge Computing for the Internet of Things," IEEE Commun. Mag., vol. 54, no. 12, pp. 22-29, 2016.

[21] X. Sun, N. Ansari, and Q. Fan, "Green energy aware avatar migration strategy in green cloudlet networks," Proc. - IEEE 7th Int. Conf. Cloud Comput. Technol. Sci. CloudCom 2015, pp. 139-146, 2016.

[22] K. S. Bahwaireth, L. Tawalbeh, A. Basalamah, Y. Jararweh, and M. Tawalbeh, "Efficient techniques for energy optimization in Mobile Cloud Computing," Proc. IEEE/ACS Int. Conf. Comput. Syst. Appl. AICCSA, vol. 2016-July, pp. 1-8, 2016.

[23] E. Benkhelifa, T. Welsh, L. Tawalbeh, Y. Jararweh, and A. Basalamah, "User profiling for energy optimisation in mobile cloud computing," Procedia Comput. Sci., vol. 52, no. 1, pp. 1159-1165, 2015.

[24] L. A. Tawalbeh, A. Basalamah, R. Mehmood, and H. Tawalbeh, "Greener and Smarter Phones for Future Cities: Characterizing the Impact of GPS Signal Strength on Power Consumption," IEEE Access, vol. 4, pp. 858-868, 2016.

[25] L. Tawalbeh, Y. Haddad, O. Khamis, F. Aldosari, and E. Benkhelifa, "Efficient software-based mobile cloud computing framework," Proc. 2015 IEEE Int. Conf. Cloud Eng. IC2E 2015, pp. 317-322, 2015.

[26] M. Al-Ayyoub, Y. Jararweh, L. Tawalbeh, E. Benkhelifa, and A. Basalamah, "Power Optimization of Large Scale Mobile Cloud Computing Systems," Proc. - 2015 Int. Conf. Futur. Internet Things Cloud, FiCloud 20152015 Int. Conf. Open Big Data, OBD 2015, pp. 670-674, 2015.

[27] Z. Xu, W. Liang, W. Xu, M. Jia, and S. Guo, "Efficient Algorithms for Capacitated Cloudlet Placements," IEEE Trans. Parallel Distrib. Syst., vol. 27 , no. 10 , pp. 2866-2880, 2016.

[28] M. Jia, J. Cao, and W. Liang, "Optimal Cloudlet Placement and User to Cloudlet Allocation in Wireless Metropolitan Area Networks," IEEE Trans. Cloud Comput., vol. 5, no. 4, pp. 725-737, 2015.

[29] Y. Mao, C. You, J. Zhang, K. Huang, and K. B. Letaief, "A Survey on Mobile Edge Computing: The Communication Perspective," IEEE Commun. Surv. Tutorials, vol. 19, no. 4, pp. 2322-2358, 2017.

[30] S. Wang, Y. Du, and Y. Deng, "A new measure of identifying influential nodes: Efficiency centrality," Commun. Nonlinear Sci. Numer. Simul., vol. 47, pp. 151-163, 2017.

[31] E. Estrada, "The communicability distance in graphs," Linear Algebra Appl., vol. 436, no. 11, pp. 4317-4328, 2012.

[32] F. Agboma, M. Smy, and A. Liotta, "QoE analysis of a peer-to-peer television system," MCCSIS'08 - IADIS Multi Conf. Comput. Sci. Inf. Syst. Proc. Wirel. Appl. Comput. 2008 Telecommun. Networks Syst. 2008, pp. 114-119, 2008. 